\title{
Art as a tool for science
}

Artistic techniques are essential tools to visualize, understand and disseminate the results of scientific research. The field of structural biology has enjoyed a particularly productive marriage of art and science.

\author{
David S. Goodsell
}

\section{$\Lambda$} $\mathrm{t}$ a recent summer internship hosted by the Djerassi Resident Artists Program, I had the opportunity to watch several fine artists at work $^{1}$. Once a year, this program tosses together six scientists and six artists for a month and provides them with the chance to create together. I was invited as one of the scientists. The experience was daunting, and in many ways, I came away with the impression that an artist's task is infinitely more difficult than the work we face as scientists. Scientists work within very tight constraints: experiments must probe the nature of the world and must be reproducible; hypotheses must account for observations in a logical way; and most importantly, scientists must then devise new ways to test and possibly destroy these hypotheses with further experimentation. Fine artists have far fewer constraints. As they create work that speaks to their audiences, they are limited only by imagination and the technicalities and seductions of their chosen media. Consequently, fine artists need to create entire worlds from scratch. My time at this internship helped me to gain a better understanding of my own artwork, where the goal is more circumscribed: to create imagery as a tool for science.

Photographer Felice Frankel beautifully articulated this goal in relation to her own work: "I do not view myself as an artist because an artist has a personal agenda and a very particular point of view-that of communicating the part of herself she wants the world to perceive. One may view the images I take as artistic, but their primary purpose is to communicate scientific information" ${ }^{2}$. The idea of borrowing the techniques of fine art for scientific communication has proven useful throughout the history of science and is currently undergoing a renaissance with the SciArt movement. The SciArt community is a wonderfully heterogeneous mix of creative people: artists working on scientific themes, scientists using art in their science, and every combination in between.

The power of SciArt has perhaps its strongest manifestation in structural biology, where the things we study are particularly amenable to visual representation. Molecules

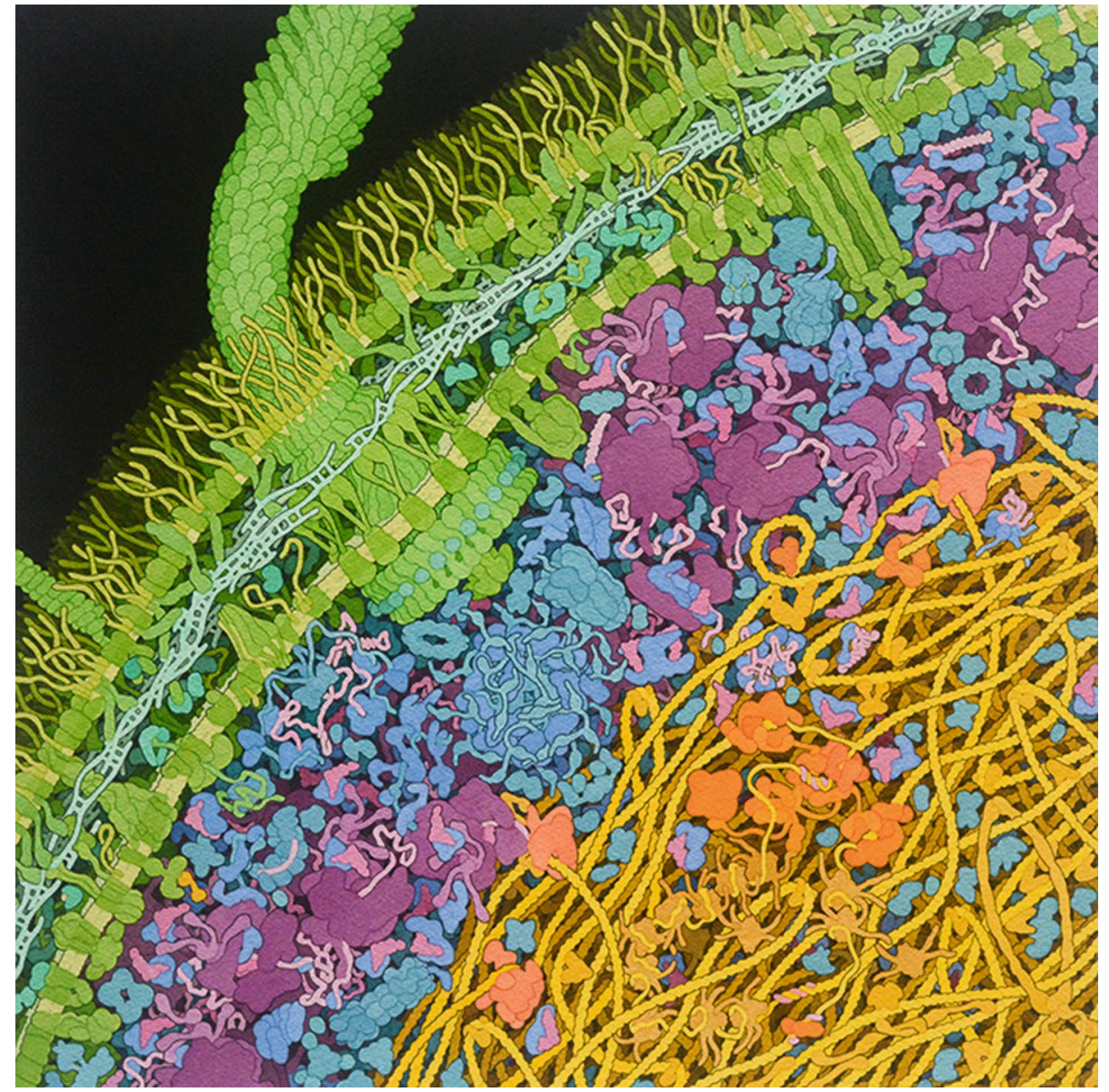

Fig. 1 | Artistic conception of a cross-section through a bacterial cell. This watercolor painting integrates information from structural biology, microscopy and bioinformatics. I explored many hypotheses during creation of the painting, which required making decisions about, for example, sieving effects of the DNA (yellow) on the distribution of soluble molecules and details of the orientation and cross-linking of peptidoglycan chains (light turquoise) in the space between the membranes. This image is available under Creative Commons at the RCSB PDB (https://doi.org/10.2210/rcsb_pdb/ goodsell-gallery-028), along with more information on what is shown.

have a size and shape, so synthetic imagery can trick us into thinking we can see them for ourselves. The early days of structural biology relied heavily on SciArt, better known at the time as "visualization." Macromolecular X-ray crystallography was one of the early drivers of computer graphics hardware and software development, and as part of this, an entire visual language was invented to depict the structure and properties of proteins and nucleic acids ${ }^{3}$.
The impact of these visual tools is hard to measure, since they are so ingrained in every aspect of our work, both in research and in its dissemination. Today, we can head over to one of the worldwide Protein Data Bank sites (https://wwpdb.org) and instantly view more than 170,000 biomolecular structures using highly sophisticated graphics tools that are available, amazingly, directly in a web browser or on your phone. As structural biologists, we're all intimately familiar with 


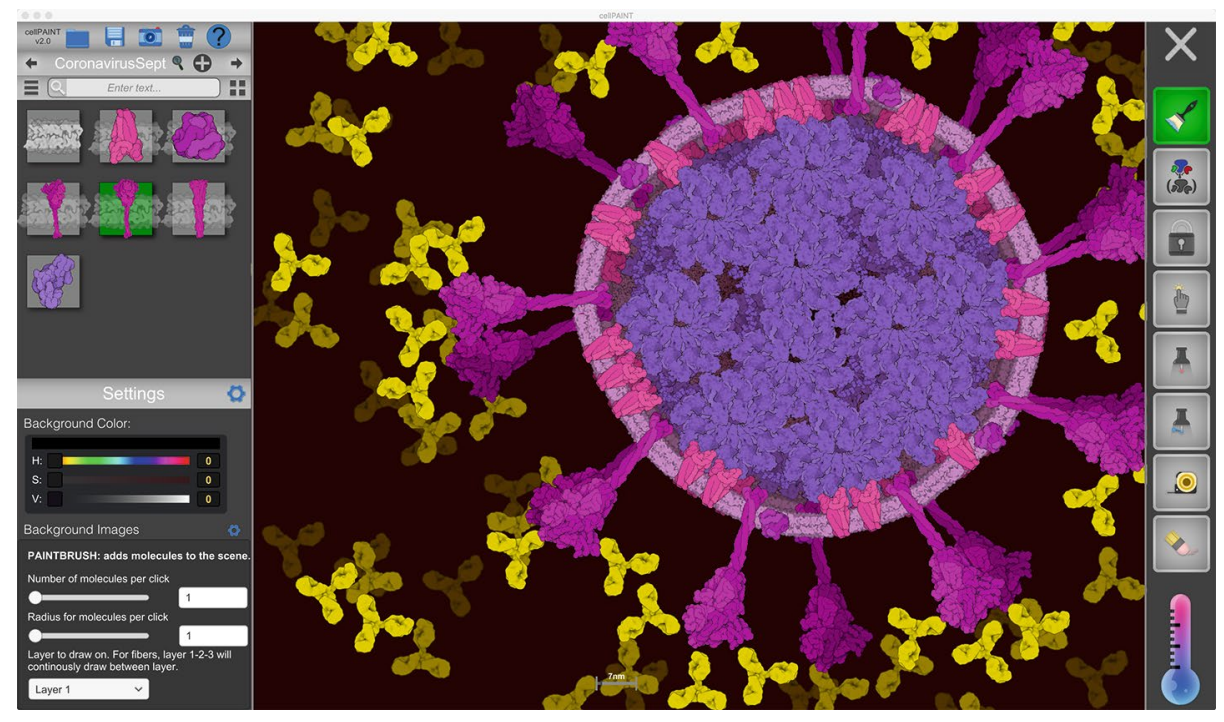

Fig. 2 | Using CellPAINT to illustrate SARS-CoV-2 surrounded by antibodies. In the digital illustration program CellPAINT (https://ccsb.scripps.edu/cellpaint), molecules are chosen from a palette on the left and painted into the scene, and various options for painting, grouping, locking and erasing molecules are available on the right. Each of the molecular brushes is controlled by the behavior of the molecule, so the spike proteins will remain embedded in the viral membrane but antibodies will be free to diffuse around the virion.

the uses of these methods. They allow us to pose structural questions on the fly and answer them interactively. We load a protein structure, measure distances and angles at coordination sites, look for neighboring amino acids and try to reconcile mutational data, color by surface charge or hydrophobicity to understand how this protein interacts with others, and so on. In my own research in computational biology and drug design, I use these tools every day without thinking twice. And when I want to present my work to other scientists or to a wider audience, I use these same tools, infused with a bit more artistic flair.

This type of SciArt-visualizationcomes with strong constraints. Visualization is a tool for study, essentially extending the capabilities of our eyes, and must be treated like any of the other materials and methods that we employ in our research. The graphical approach needs to capture the salient properties of the molecule so that the insights we gain during the visualization will translate into insights about the biology. When used as figures in our papers, these images are documentary evidence of our discoveries and thus require a direct connection between the data and image, with no cherry-picked image processing or manual tweaking. To me, the constraints of scientific visualization are far more a joy than they are a curse. They invite me to focus on the goals of the image, and once these goals are set, I can leverage the creativity that we borrow from fine art to refine and simplify the visual method until it perfectly captures the desired properties of the molecule.

SciArt can also help us to see the larger context of our work. Artistic conceptions provide an easy way to explore speculative hypotheses about how our data fit together into a big picture. When constrained with a scientific sensibility, this is a powerful tool for synthesizing an increasingly comprehensive representation of the data to act as a touchstone for future thought and research. Speculative SciArtists continually ask difficult questions like this to explore unfamiliar worlds: Chesley Bonestell imagined what we would see if we stood on the surface of Titan; Isaac Asimov asked what it would be like to journey through the bloodstream. We can take this same approach as a scientific tool in structural biology.

In my postdoctoral work, I asked myself the question: "Can I paint an accurate picture of the molecular structure of a living cell?" After many hours in the library with the citation index and much enjoyable exploration of the Protein Data Bank (at the time, 700-entries large!), my answer was "Almost." With a liberal dose of artistic license and scientific intuition, I cobbled together as much information as I could find into an image of a portion of a bacterial cell ${ }^{4}$. This process was filled with hypotheses that needed answers: What direction do the peptidoglycan strands go? How bendy and supercoiled is the DNA? When RNA polymerase moves down the helical DNA strand, does the nascent mRNA end up wrapping around the DNA? In the years since then, as more and more structural, proteomics and ultrastructural data have become available, I have continued to update and refine this image (Fig. 1).

The process of creating this type of integrative image, rather than the final image itself, is arguably the most important aspect of the endeavor. This is when the fun begins, as it involves searching for information from multiple disciplines, fitting it together to build a larger picture, and filling the gaps with best guesses. I have since worked with many researchers to create similar integrative illustrations based on their work (see for example work on depicting autophagy with Daniel Klionsky ${ }^{5}$ ). Invariably, the researchers learn as much as I do as we gather information on the parts of the painting related to their work, as well as information about the many other details that need to be included: the cellular context of their molecular work, or the molecular details of their cellular work.

In my laboratory, we are building software to help researchers create these types of integrative conceptions of their own work without the need for art classes and hours of painting. CellPAINT (Fig. 2) allows researchers to build up cellular illustrations that are similar to my paintings, using a set of molecular brushes that have molecule-like behaviors $^{6}$. The goal is to put more tools into the hands of scientists, thereby reducing the barrier between their ideas and the manifestation of these ideas in images. In addition, by simplifying and streamlining the process of building these types of integrative illustrations, we can help in keeping up with the steady forward march of science. I always joke that my paintings go out-of-date the second I finish them. But that is the power of SciArt: it captures the current state of knowledge, warts and all, and hopefully spurs discussion and further exploration.

\section{David S. Goodsell (iD) 1,2四}

${ }^{1}$ Department of Integrative Structural and Computational Biology, the Scripps Research Institute, La Jolla, CA, USA. ${ }^{2}$ Research Collaboratory for Structural Bioinformatics Protein Data Bank,

Rutgers, the State University of New Jersey,

Piscataway, NJ, USA.

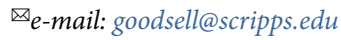

Published online: 7 May 2021 https://doi.org/10.1038/s41594-021-00587-5

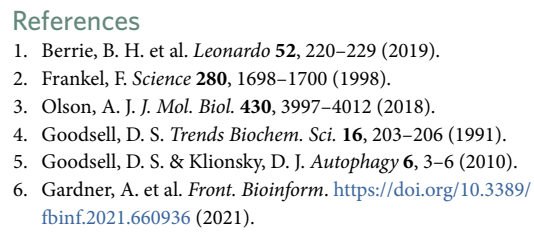

The author declares no competing interests. 